\title{
Física para simples mortales
}

\section{Joanne Striley}
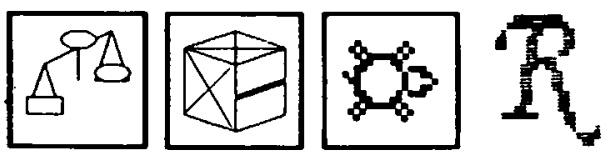

La comprensión de las relaciones y leyes físicas depende, como se analiza en este artículo, del modo en que se represente éstas el alumno. El predominio de sistemas matemáticos de explicación e intuición de las leyes físicas -la vía cuantitativa- parece producir una visión opaca de esas leyes y lleva a errores graves de comprensión, pese a la correcta, pero inestable aplicación puntual de fórmulas.

En este artículo se exponen nuevos métodos de enseñanza de la fisica por ordenador que presentan entornos interactivos cuasi-reales - micromundos-y que se basan en sistemas visuales -via cualitativa- baciendo asi transparentes los procesos fisicos tradicionalmente ocultos tras el ropaje notacional de los operadores matemáticos.

Los alumnos del último año de enseñanza secundaria habían terminado su curso de Física, la mayoría con éxito. En el examen final la inmensa mayoría de los alumnos había calculado correctamente la velocidad y la posición de objetos móviles en diversas situaciones. Sin embargo, cuando se les pidió que describiesen la trayectoria de una pelota lanzada desde un acantilado, muchos de ellos indicaron que la pelota se desplazaría en línea recta a partir del acantilado y paralelamente al suelo durante algún trecho y que después caería recta (en picado), un hecho no muy infrecuente en los dibujos animados de "Correcaminos» (Road Runner), pero que transgredía las leyes del movimiento que los estudiantes acababan de utilizar también en sus cálculos. Al igual que muchos buenos estudiantes de Física, estos alumnos demostraron que podían aplicar las fórmulas y realizar cálculos para situaciones que comprenden esencialmente mal. Mientras, y al otro lado de la ciudad, un grupo de estudiantes de primaria de sexto realizaba el mismo grupo de problemas. Estos alumnos demostraron ser tan competentes en calcular las velocidades y posiciones como sus colegas más mayores, pero cuando se les pidió que dibujasen la trayectoria de la pelota lan-

«Physics for the Rest of Us.» Educational Researcher. Agosto-septiembre 1988, pp. 7-10. Reproducido con autorización. (CTraducción al castellano, CL\&E, 1989 (traducción de Carmen Trillo). 
zada desde el acantilado, la mayor parte de los alumnos de sexto dibujaron un arco correcto.

Lo que los estudiantes de sexto curso hicieron y los estudiantes del $12 .^{\circ}$ curso (COU) no supieron hacer fue razonar cualitativamente sobre la mecánica Newtoniana. Esta capacidad les permitió analizar situaciones que constituyen auténticas trampas para los estudiantes de Física. ¿Qué ocurre si dos pelotas se lanzan desde el acantilado al mismo tiempo, una con un golpe fuerte y otra con un toque suave? En el modelo del Correcaminos la pelota golpeada fuertemente se desplaza en línea recta desde el acantilado mientras que la otra pelota cae directamente hacia abajo. Este modelo brinda la predicción habitual de que las pelotas golpearán el suelo en tiempos distintos. La mayoría de los estudiantes tienen que realizar muchos cálculos para darse cuenta de que las pelotas realmente tocan el suelo a la vez. Los estudiantes de sexto curso fueron capaces de aplicar el razonamiento cualitativo para encontrar la respuesta correcta sin recurrir a los cálculos: dado que la fuerza de la patada y la atracción de la gravedad opera en cada pelota simultáneamente (y no una detrás de la otra, como en el modelo del Correcaminos) la pelota golpeada con más fuerza aterrizará más lejos del acantilado pero al mismo tiempo que la pelota golpeada con menos fuerza.

La ventaja de los estudiantes de sexto curso se explica porque habían seguido un sistema de enseñanza de la mecánica basado en el ordenador y denominado «Instrumentos para Pensar» (Thinker-Tools). Bárbara White y Paul Horwitz de los laboratorios BBN de Cambridge, Massachusetts, los diseñadores del sistema, forman parte del creciente número de investigadores que emplean software para investigar problemas conceptuales en el aprendizaje de las ciencias. Mediante una cuidadosa secuencia de actividades encaminadas a la resolución de problemas a modo de juego, los estudiantes pueden utilizar el sistema para explorar modelos de movimiento y adquirir gradualmente comprensión cualitativa.

\section{CAMBIANDO LA DIRECCION DE LA ENSEÑANZA}

Los estudiantes de $12 .^{\circ}$ curso cuyos dibujos semejaban más los de una dibujante de comics que los de un científico, son bastante representativos, tanto de los estudiantes universitarios (college) como de los estudiantes de enseñanza secundaria. Durante los últimos años, la investigación sobre las concepciones científicas erróneas ha comenzado a sondear las profundidades de esas dificultades que tienen los estudiantes para aprender principios científicos contra intuitivos, y la mayor parte de este trabajo se ha centrado en física elemental. Un estudio tras otro han evidenciado que la comprensión del estudiante sólo forma una fina capa de competencia cuantitativa que se desprende fácilmente revelando un núcleo de ideas intuitivas y acientíficas. Al igual que nuestros estudiantes de $12 .^{\circ}$ curso, los alumnos son capaces de aplicar de forma típica ecuaciones para obtener respuestas numéricas a determinados tipos de problemas, pero no muestran ninguna comprensión de lo que físicamente significan o suponen esas ecuaciones.

También se ha demostrado que esta falta de comprensión cualitativa obstaculiza a la larga el rendimiento cuantitativo. Desde el momento en 
que los estudiantes se aventuran más allá de los típicos problemas incluidos al final de la lección y claramente ajustados a los ejemplos del texto, ya no son capaces de decir qué ecuaciones deberían aplicarse para encontrar la solución. Las investigaciones realizadas para establecer las diferencias entre expertos (físicos universitarios) y novatos (estudiantes de física elemental) señalan el papel crucial que desempeña la comprensión cualitativa. Cuando se enfrenta con un problema nuevo, normalmente el experto realiza primero un análisis cualitativo para hacerse un idea del tipo de problema que tienen entre manos y aplica después las ecuaciones sugeridas por el análisis para delimitar el cuadro cualitativo y emitir una solución numérica. El novato, por el contrario, intenta hacer un análisis cuantitativo en primer lugar, considerando todas las posibles ecuaciones que podrían aplicarse. Sólo entonces, y, para salir de este mar de fórmulas, el novato extrae ideas cualitativas mal elaboradas, como el modelo de movimiento del Correcaminos. El novato no sólo intenta hacer que el carro conduzca al caballo, por decirlo así, sino que tampoco es consciente de los aspectos fundamentales del sistema: las ruedas del carro, los arreos del caballo, etc.

La enseñanza de la Física puede alentar esta falta de consciencia mediante el gran énfasis que suele dar al trabajo cuantitativo. La mayor parte de los investigadores estarían de acuerdo con Hermann Haertel, del Instituto de Ciencias de la Educación en Keil, República Federal Alemana, cuando afirma que: «el método actual para enseñar física es bueno para manejar las abstracciones en forma de anotación matemática, pero es débil a la hora de apoyar el pensamiento cualitativo de forma consistente." La excesiva confianza en procedimientos cuantitativos y en formulismos matemáticos favorecida por la enseñanza de las ciencias físicas se manifiesta en lo que a los profesores de ciencias les gusta denominar «el síndrome del licenciado»: un estudiante al que se le pide durante exámenes eliminatorios que senale hacia el cielo, empezará inmediatamente a garabatear ecuaciones.

Puede parecer algo irónico utilizar ordenadores, las máquinas cuantitativas más recientes, para favorecer la comprensión cualitativa. Sin embargo varios equipos de investigación están trabajando con sistemas basados en el ordenador diseñados para lograr ese propósito y que soportan la compleja carga computacional inherente a la física cuantitativa, dejando por tanto al estudiante libre para concentrarse en la exploración de las relaciones cualitativas fundamentales. En palabras de Ruth Chabay de la Universidad de Carnegie-Mellon (CMU): «no vamos a ignorar los aspectos cuantitativos de la física, pero vamos a dejar que los ordenadores hagan los cálculos. Computar, después de todo, es lo que los ordenadores hacen mejor.» $\mathrm{Al}$ igual que en «Instrumentos para pensar» (Thinkier Tools) de White y Horwitz, el ordenador puede modelar para el estudiante situaciones físicas, mediante simulaciones interactivas o micromundos. Chabay describe un pequeño prototipo elaborado en el centro para el Diseño de informática Educativa de la mencionada universidad (CDEC). En él se muestran dos partículas en la pantalla de un ordenador, una en un sitio fijo del centro, y otra que es móvil y se controla con el ratón del ordenador. Conforme el usuario desplaza el objeto libre, el ordenador calcula las fuerzas aplicables en ambos objetos en cada instante. Sobre la pantalla los vectores acoplados a los objetos cambian continuamente de longitud y de dirección para demostrar las fuerzas en tiempo real. Un menú permite al usuario explorar 
las ramificaciones de ese sistema bajo diferentes leyes de atracción entre las dos partículas: ¿qué ocurre si la atracción es proporcional a dos veces la distancia entre los objetos? ¿o al cuadrado de la distancia? Al realizar experimentos en estos micromundos, los estudiantes pueden desarrollar intuiciones cualitativas sobre cómo se comportarán distintos sistemas bajo distintas circunstancias. La demostración del ordenador también ayuda a los estudiantes a comprender y pensar en términos de entidades abstractas como vectores de fuerza, y a construir los modelos físicos causales que subyacen tras el comportamiento de los sistemas.

Un enfoque también relacionado con la ayuda mediante ordenadores a la comprensión cualitativa y que lleva actualmente a cabo Robert Tinker en los Centros de Investigación en Educación Técnica (TERC) en Cambridge, consiste en hacer que los estudiantes trabajen con fenómenos reales en un laboratorio práctico tradicional, pero que utilicen el ordenador para la recogida de datos y el análisis y para ensayar diferentes modelos explicativos.

\section{CUMPLIENDO LA PROMESA DEL ORDENADOR}

Aunque los investigadores han anticipado ya hace largo tiempo la promesa que constituían las simulaciones en ordenador para la educación, la realización de esta promesa parece que tarda en conseguirse. Jill Larkin, que dirige el CDEC, atribuye este retraso en parte a «la tendencia de los investigadores universitarios a trabajar con sofisticadas máquinas de vanguardia que pueden proporcionar rasgos como gráficos de elevada calidad, ventanillas que aparecen inesperadamente (Pop - ups windows), y control del ratón. Estos ambientes son poderosos y de un uso atractivo, pero también son caros y difíciles de programar». A este problema se añade, destaca ella, la incompatibilidad general de distintos tipos de máquinas. Al igual que los pinzones de Darwin, los programadores que trabajan con diferentes tipos de ordenadores dan origen a líneas de descendencia que no son fecundas por fertilización cruzada. Por tanto, el esfuerzo de investigación y desarrollo es lento y fragmentado, y los prototipos de micromundo desarrollados en las universidades y centros de investigación raramente encuentran su camino hasta las escuelas.

El grupo de investigación de Larkin ha desarrollado un entorno de programación que piensan pueda ayudar a vencer estas dificultades. El cT (en un principio Tutor del CMU) como se denomina el sistema, proporciona acceso a aplicaciones sofisticadas además de una base para nuevos programadores. "La meta es proporcionar un medio flexible, portatil y no obstante poderoso, para la programación instruccional que tanto los educadores como los programadores profesionales puedan utilizar», dijo Larkin. El cT correrá en diversas redes muy extendidas, así como en los microordenadores Apple Macintosh y PC de IBM, de forma que el sofware desarrollado con máquinas grandes pueda distribuirse directamente a las escuelas.

Aunque el entorno del cT se está utilizando en diversas universidades con aplicaciones para la investigación y el desarrollo, el CDEC trata de probar su potencial utilizando el c'T para crear software que apoye la enseñanza cualitativa y rigurosa de la Física en la enseñanza secundaria en niveles 
universitarios elementales. El grupo intenta hacer desarrollos instruccionales de temas elementales, como el electromagnetismo, para los que las matemáticas necesarias a menudo se encuentran más allá del alcance de incluso los estudiantes principiantes más capaces. «En lugar de pasar por alto los temas o de cargar a los estudiantes con procedimientos matemáticos que no comprenden y que confunden la Física, se dará a los estudiantes micromundos para explorar que les ayuden a llegar a serias nociones cualitativas» dice Larkin. "Preferimos tener estudiantes principiantes que sean capaces de razonar que una partícula situada en un campo eléctrico experimentará una fuerza en una dirección determinada y que, por consiguiente, se desplazará incialmente en esa dirección, por ejemplo, que hacer que esos estudiantes traten de analizar las situaciones mediante ecuaciones.»

El trabajo actual en el grupo del CDEC se centra en el perfeccionamiento del entorno del cT y el diseño instruccional. Pruebas concretas sobre el currículum tendrán lugar en el curso de Física elemental para adolescentes el próximo año. Según Bruce Sherwood, del CDEC, y miembro de la facultad de Física de la CEMU, esta integración curricular distingue al proyecto de otros esfuerzos anteriores sobre simulaciones de ordenador diseñadas para reificar conceptos físicos abstractos. «Normalmente dichos programas se presentan como una pantalla casi en blanco donde se invita a un estudiante a, digámoslo así, teclear un número que representa la velocidad a la que se chuta una pelota de fútbol, y el programa de simulación muestra cómo la pelota describe un arco en el aire. A continuación, el estudiante puede teclear un número diferente para una velocidad diferente, etc. El problema con simulaciones crudas de es clase es que la mayor parte de los estudiantes principiantes no tiene ninguna pregunta que hacer, y sin un contexto que proporcione algunas metas, es frecuente que los estudiantes no obtengan casi nada de estas simulaciones. Hay un elevado número de ejemplos de simulaciones de ese tipo, pero muy pocos intentos por desarrollar una cobertura completa del curso en que los materiales del ordenador estén incluidos en un marco instruccional coherente.»

En otra parte de Pittsburgh, Kalyani Raghavan, Jamie Schultz y Leona Schauble están trabajando con Robert Glaser para diseñar un sistema de autor que ayude a los profesores a construir para sus alumnos entornos de descubrimiento basados en el ordenador. Un micromundo prototipo permite a los estudiantes diseñar experimentos con simples circuitos sencillos de corriente continua (Direct Current). La idea clave de este trabajo es enseñar destrezas de autointerrogación. En las investigaciones de evaluación, del programa los estudiantes generaron datos satisfactoriamente y utilizaron las herramientas de manejo de datos del ordenador para descubrir las leyes de los circuitos de Ohm y de Kirchoff. "Comprobamos que los programas de tratamiento de datos que habíamos desarrollado eran instrumentos genéricos que podían ayudar a enseñar estrategias de aprendizaje por descubrimiento en muchos ámbitos» afirma Raghavan, «por lo que estamos ideando un sistema de autor que permita a los expertos integrar sus contextos de ámbito específico con estas herramientas generales mediante una programación mínima». 


\section{SIMULANDO LOS FENOMENOS}

El rigor es un aspecto importante a la hora de adoptar un enfoque cualitativo. Pero en la construcción de modelos sobre fenómenos es inevitable alguna distorsión. El único fenómeno semejante a la electricidad en todos los aspectos es la propia electricidad; un diagrama de simulación siempre miente de alguna manera sobre lo que representa. ¿Cómo afecta esta impresión al aprendizaje y a la comprensión? ¿Ayuda el interactuar con un micromundo a erradicar falsas concepciones o podría en realidad crearlas?

$\mathrm{La}$ evidencia de que las analogías influyen en las pautas de inferencia de los estudiantes recalca esta preocupación. Deidre Gentner, de la Universidad de Illinois, ha examinado dos populares analogías del comportamiento del circuito eléctrico: una compara la conducta del circuito de corriente continua con el agua que fluye a través de tuberías y válvulas; la otra con una multitud de gente empujando atropelladamente por los pasillos hacia las salidas. Gentner se encontró con que los estudiantes universitarios y de enseñanza secundaria tenían concepciones y falsas concepciones distintas, según la analogía que utilizasen para analizar los circuitos de corriente continua. Por tanto, la elección del modelo es, en potencia, bastante importante.

Hermann Haertel rechaza tanto la analogía de flujo de agua como de torrente de gente cuando desarrolla su enfoque cualitativo para la enseñanza de la electricidad, puesto que considera esas analogías imprecisas y erróneas en diversos puntos clave. La multitud, por ejemplo, se compone de individuos con sus propios dispositivos independientes de locomoción, lo que les da propiedades muy diferentes, manifiesta, de las de los electrones de un circuito. Haertel destaca que estas populares analogías podrían utilizarse en la enseñanza como ejemplos negativos. Hacer explícitas sus imprecisiones sería una forma de apuntar a modelos físicos del circuito más correctos.

Los modelos cualitativos de Haertel de los fenómenos eléctricos utilizan el concepto de carga superficial y tratan con representaciones cualitativas de los campos y de la propagación de las ondas. Cuando la secuencia de materiales interactivos de aprendizaje que ha de sevir de ayuda esté a punto, Haertel probará estos modelos con los estudiantes. Otros investigadores piensan que, al intentar ser riguroso, Haertel puede haber creado modelos que resulten demasiado complejos y formales para el uso práctico de los estudiantes. John Fredericksen de BBN, piensa que la búsqueda de modelos completamente precisos es de alguna manera ilusoria. En opinión de Fredericksen, Haertel simplemente ha empujado la aproximación hacia un nivel anterior al concepto de carga de superficie.

Fredericksen y White están desarrollando actualmente sus propios modelos para circuitos de corriente continua. Su idea es proporcionar una progresión de modelos de complejidad creciente conforme el estudiante analiza circuitos cada vez más elaborados. Los modelos implementan simulaciones de circuito (representadas mediante diagramas en la pantalla del ordenador) y utiliza el habla del ordenador para guiar a los estudiantes a través del análisis del circuito. Se han basado para ello en las observaciones realizadas por White y Fredericksen sobre la labor de un profesor de enseñanza secundaria con auténtico talento que enseñaba a sus estudiantes 
cómo realizar análisis cualitativos de circuitos como el primer paso a seguir para resolver cualquier avería electrónica.

En una evaluación preliminar de este programa QUEST (Qualitative Understanding of Electrical System Troubleshooting) con estudiantes de enseñanza secundaria, la capacidad de los estudiantes para investigar los problemas de los circuitos mostró un destacado incremento tras cinco horas de trabajo con los modelos cualitativos. Los estudiantes que en un principio carecían de cualquier noción funcional sobre un circuito eléctrico (p.e., los que pensaban que una simple conexión entre la pila y la bombilla sería suficiente para encender la bombilla) resolvieron con éxito todos los problemas de predicción y solución de conflictos del circuito en un posttest.

Sin embargo, no hay nada en los modelos QUEST que haga explícita la física subyacente. Guiados por los modelos, los estudiantes aprenden a razonar que cerrar un interruptor en un circuito origina una caída de voltaje a través de una bombilla, pero no tienen ninguna forma de razonar sobre lo que es ese voltaje o cómo se distribuye a través del circuito en términos de conceptos básicos de materia y energía. Aunque empleada muy raramente por los técnicos de avería esta perspectiva reduccionista, que busca vincular fenómenos de nivel macro y micro, es una meta importante para la educación en ciencias. Para desarrollar su sistema, White y Fredericksen están trabajando con un conjunto de modelos físicos que relacionan el votaje con conceptos mecánicos.

Aunque algunos manifiestan que los modelos físicos reduccionistas no son necesarios y otros piensan que son necesariamente inexactos $y$, como tales, una posible fuente de falsas creencias, Bárbara White cree que los modelos físicos de causalidad básica, al proporcionar un marco explicativo adicional, pueden ayudar a los estudiantes a comprender mejor los modelos fenomenológicos. "Creo que sería deseable proporcionar a los estudiantes diversas clases de modelos, cualitativos, cuantitativos, físicos, funcionales, y conductuales, a los que se pueda acudir en distintas situaciones", afirma, "La gente necesita adquirir modelos de distinto tipo antes de que comprenda algo en profundidad y sea capaz de resolver un amplio abanico de problemas. Ahora la pregunta que surge es: ¿en qué orden?, ¿en qué progresión? En un principio defendimos que deberiamos comenzar con modelos conductuales cualitativos y a partir de ahí pasar hacia modelos cuantitativos, pero ahora pensamos que sería mejor presentarlos simultáneamente. El aspecto de progresión tiene que ver con la capacidad de aprendizaje, con lo que el estudiante está construyendo y la clase de comprensión que está elaborando".

\section{APLICANDO EL ENFOQUE CUALITATIVO: QUIEN, QUE, CUANDO}

La información que debería incorporar un modelo y la secuencia en la que presentar distintos modelos de instrucción son asuntos candentes en investigación educativa. Uri Ganiel y Bat-Sheva Eylon del Instituto Weizmann de Rehovot, Israel, manifiestan que para entender los circuitos de corriente continua en los niveles de enseñanza secundaria o universitaria elemental, las relaciones cualitativas y las relaciones macro-micro de causali- 
dad física son todas importantes, pero que cada una tiene un rango distinto de aplicación en el currículum. Ellos creen que el análisis cualitativo es práctico sólo para circuitos relativamente simples, mientras que «el análisis cuantitativo sigue a algoritmos bien definidos y así puede aplicarse incluso a circuitos complejos».

En opinión de Larkin los aspectos cualitativos son más cruciales para los estudiantes principiantes. «Para estudiantes de enseñanza secundaria y de especialidades de humanidades universitarias, no encuentro ninguna razón para enseñarles todos los difíciles aspectos cuantitativos de temas como el electromagnetismo. Si la enseñanza constituyese parte de un curso para especialistas en física se podría continuar a lo cuantitativo." La cuestión, cree ella es que «los cursos actuales presentan un fuerte énfasis cuantitativo, y queremos que esto cambie radicalmente».

Andrea di Sessa de la Universidad de California, Berkeley, sugiere que la línea entre lo cualitativo y lo cuantitativo puede estar excesivamente marcada: «En mi opinión, lo cualitativo representa un grito de ánimo para hacer que cambiemos completamente, pero no dice mucho sobre qué camino tomar.»

Si los estudiantes no encontrasen las matemáticas avanzadas tan difíciles, veríamos menos clara una distinción precisa entre físicas cuantitativas y cualitativas. El enfoque adoptado por Robert Pinker del TERC está más próxima, en cierta medida, a modelar las matemáticas que a modelar el sistema físico. En lugar de hacer que una simulación por ordenador presente algún modelo del medio ambiente físico, los laboratorios basados en microordenadores (MBL), de Tinker ayudan a la recolección, exhibición y análisis de datos experimentales reales. Mediante la transformación de datos brutos en manifestaciones gráficas en tiempo real sobre la pantalla del ordenador de un Apple II, el MBL ha proporcionado, incluso a los estudiantes de nivel elemental, una comprensión fenomenológica seria de, por ejemplo, los cambios de temperaturas asociados con los cambios de estado.

Sin embargo, al igual que en el estudio QUEST de White y Fredericksen, Tinker se ha encontrado con que esta comprensión a nivel macro no provoca necesariamente una comprensión de la física subyacente. Por ello ha venido trabajando en el diseño de una herramienta para construir modelos con la que los estudiantes pueden construir modelos matemáticos manipulando símbolos gráficos en la pantalla del ordenador. Estos símbolos gráficos se combinan para representar modelos causales de la conducta de sistemas dinámicos. En los primeros ensayos con el sistema de enseñanza secundaria construyeron y analizaron modelos de procesos tales como el flujo de fluidos o la descarga del condensador, que ordinariamente habrían estado representados por grupos de ecuaciones diferenciales.

\section{ABRIENDO LA PUERTA}

El enfoque cualitativo para la enseñanza de la Física presenta el potencial necesario para ayudar a encauzar dos importantes problemas. Por una parte al proporcionar a los alumnos de especialidades en ciencias una comprensión más completa de cómo se comportan los sistemas físicos, puede ayudar a cimentar el aprendizaje de las ecuaciones físicas sobre nociones 
físicas correctas, en lugar de dejar que las ecuaciones entren en conflicto con las concepciones ingenuas de los estudiantes. Sustituir estas concepciones ingenuas por otras bien fundadas deberían promover un avance mejor y más rápido hacia conocimientos técnicos. Y por otra parte, sacando a la Física del campo de las matemáticas y acercándola más a la experiencia cotidiana, el enfoque cualitativo puede lograr abrir el campo de la Física todos nosotros, al resto de los mortales.

\section{Referencias}

Haertel, H. (1987). A Qualitative Approach to Electricity (Tech. Rep.) Palo Alto, CA: XEROX PARC, Institute for Research on Learning.

SHERWOOD, B. A., y LARKIN, J. H. (1986). New tools for courseware production. Proceedings of the IBM Academic Informations Systems University AEP Conference. Boston, MA.

WHITE, B. Y., y FREDERICKSEN, J. R. (1987). Causal model progressions as a foundation for intelligent learnign environments. (Tech. Rep. No. 6686). Cambridge, MA: Bolt Beranek and Newman Laboratories.

ZUMAN, J. P., y WEAVER, S. L. (1988). Tools for teaching problem solving: An evaluation of a modeling and systems thinking approach. (Tech. Rep.). Cambridge, MA: Technical Education Research Centers.

\section{Datos sobre la autora}

Joanne Striley trabaja en los problemas de la enseñanza de las ciencias como doctora miembro del Centro para el Desarrollo de la Investigación en Aprendizaje, en la Universidad de Pittsburgh. 


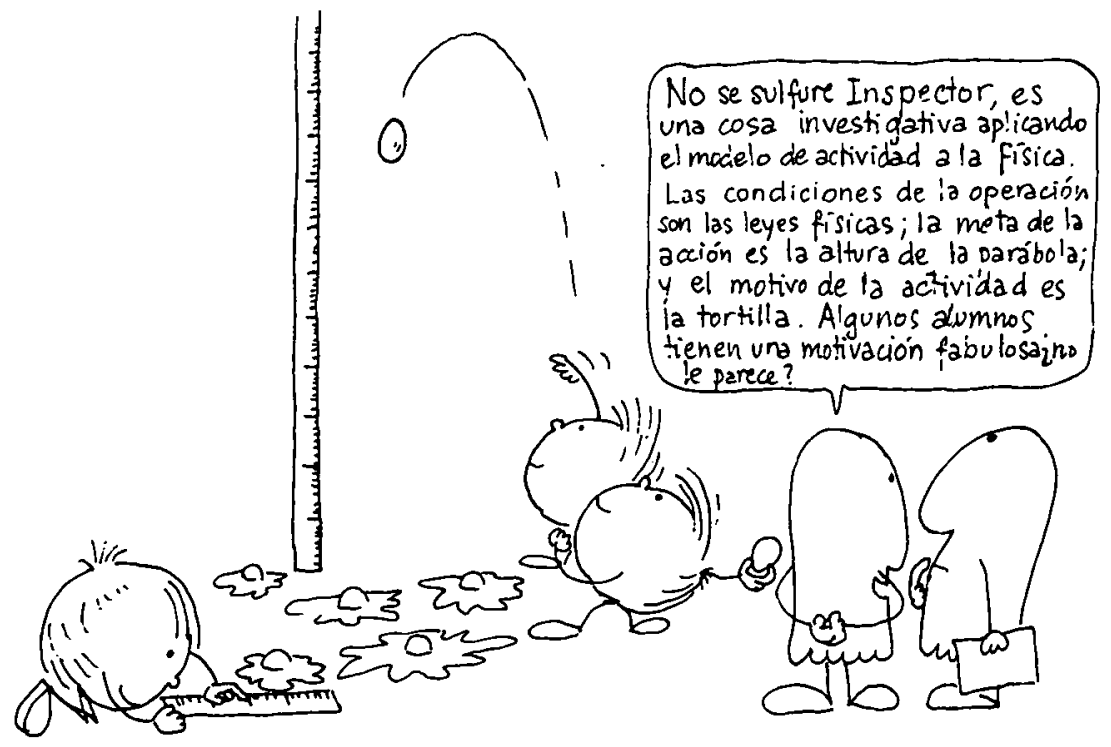

